\title{
A Framework to Explore Workload-Specific Performance and Lifetime Trade-offs in Neuromorphic Computing
}

\author{
Adarsha Balaji, Shihao Song, Anup Das, Nikil Dutt, Jeff Krichmar, \\ Nagarajan Kandasamy, Francky Catthoor
}

\begin{abstract}
Neuromorphic architectures with non-volatile memory (NVM) implement biological neurons and synapses to execute spiking neural networks (SNNs). To access synaptic weights, an NVM cell's peripheral circuit drives current through the cell using a high bias voltage, generated from an on-chip charge pump. High-voltage operations induce aging of CMOS devices in the charge pump, leading to negative bias temperature instability (NBTI) and hot carrier injection $(\mathrm{HCl})$ generated defects. Therefore, charge-pump aging poses a significant threat to the operating lifetime of neuromorphic architectures. Discharging a stressed charge pump periodically can lower its aging rate, but makes the architecture unavailable to process spikes while its charge pumps are being discharged. This introduces delay in spike propagation, which impacts inter-spike interval (ISI), leading to information loss and challenging the integrity of SNNs. This performance and lifetime trade-off depends on the SNN workload being executed. In this paper, we propose a novel framework to exploit workload-specific performance and lifetime trade-offs in neuromorphic computing. Our framework first extracts the precise times at which spikes are generated on all synapses of a SNN workload. This timing information is then used wihtin a new analytical formulation to estimate aging of charge pumps based on the SNN's mapping to the hardware and the power delivery architecture of charge pumps. We use the developed framework to optimize the mapping of neurons and synapses at design time and to schedule the discharge of stressed charge pumps at run time to maximize their lifetime, without significantly hurting the workload's performance.
\end{abstract}

Index Terms-Neuromorphic computing, Non-voltaile Memory (NVM), Phase-Change Memory (PCM), wear-out, Negative Bias Temperature Instability (NBTI), Spiking Neural Networks (SNNs), and Inter-Spike Interval (ISI).

\section{INTRODUCTION}

$\mathrm{N}$ EUROMORPHIC architectures such as TrueNorth [1], Loihi [2], and DynapSE [3] implement biological neurons and synapses to efficiently execute machine learning techniques with spike model and brain inspired learning algorithms, e.g., spiking neural networks (SNNs) [4]. A typical neuromorphic architecture consists of artificial neurons, which generate spikes when a neuron's action potential exceeds a threshold and crossbars with non-volatile memory (NVM) cells, which store synaptic weights [5]. Each crossbar has a peripheral circuit to drive current for reading and programming synaptic weights based on spikes generated from neurons in the architecture.

To access, i.e., read and program an NVM cell in a crossbar, its peripheral circuit drives current through the cell using a bias voltage generated from an on-chip charge pump. This voltage must be high enough to compensate for IR drop and the built-in potential of the access device, which connects the cell to a row and a column in the crossbar.

High-voltage operations are common in neuromorphic architectures with PCM, FeRAM, and Flash NVMs, and they are generated via on-chip charge pumps built using CMOS devices [6]. Unfortunately, when a CMOS device

- A. Balaji, S. Song, A. Das, N. Kandasamy are with the Department of Electrical and Computer Engineering, Drexel University, Philadelphia, PA, 19104 USA E-mail:anup.das@drexel.edu.

- N. Dutt and J. Krichmar are with the Department of Computer Science, University of California, Irvine, CA, XXXXX USA.

- F. Catthoor is with Imec, Belgium and KU Leuven, Belgium.

Manuscript received Month DD, YYYY; revised Month DD, YYYY. is stressed to a high voltage for a long duration, aging effects start to accelerate in the device, leading to negative bias temperature instability (NBTI) and hot carrier injection (HCI) generated defects [7]. These are the dominant ones for scaled technology nodes. In older nodes, other defects such as time-dependent dielectric breakdown (TDDB) still play a role. Aging-induced defects depend on the neural activity in the SNN workload being executed on the hardware and they lower the lifetime of CMOS devices in charge pumps.

Aging-induced defects are different from endurance failures, which are due to repeated programming of NVM cells. In neuromorphic computing, aging issues arise during inference (reading of synaptic weights) and training (update of synaptic weights) in supervised machine learning, while endurance issues arise only during training. So, ensuring long lifetime of charge pumps is critical because a faulty charge pump cannot provide bias voltages to crossbars, making those crossbars unusable for SNN execution.

To reduce the rate of aging, stressed charge pumps can be discharged periodically, where a discharge operation involves applying a low voltage to all CMOS devices in the charge pump. Once discharged, the charge pump requires several cycles to boost its voltage back, before it can safely be used to power peripheral circuits. During this interval, the neuromorphic architecture is unable to process spikes, introducing a spike propagation delay, which directly impacts the performance (e.g., accuracy) of the SNN being executed.

This paper develops a novel framework to evaluate performance and lifetime trade-offs in neuromorphic computing. The framework incorporates the CARLsim simulator [8] to first extract the precise time of neural activity in a network 


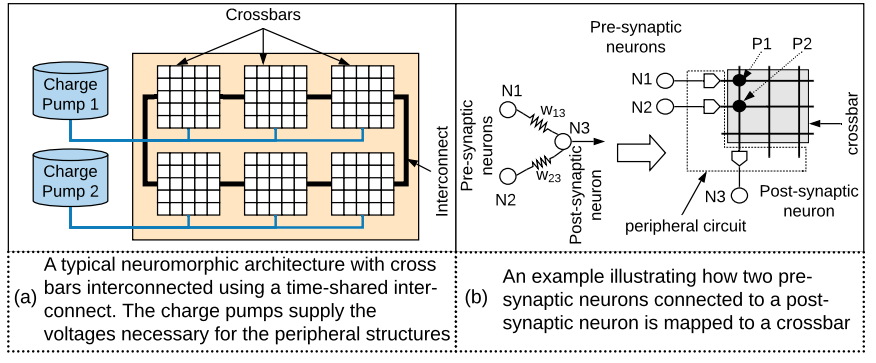

Fig. 1: An illustration of a typical neuromorphic architecture and how SNNs are mapped to a crossbar in this architecture.

of spiking neurons in response to stimuli applied at its input for a given workload. We then propose a new analytical model that uses this spike timing to estimate aging in charge pumps based on the synapse-to-crossbar mapping and power delivery architecture of charge pumps. We show that this framework can be integrated inside 1) designtime based techniques, where neurons and synapses can be efficiently allocated to different crossbars, balancing aging of all charge-pumps, 2) run-time based techniques, where stressed charge pumps can be discharged at appropriate intervals, minimizing their aging without significantly impacting performance, and 3) architectural techniques, where a charge pump's power delivery architecture can be optimized to achieve a target lifetime.

\section{BACKGROUND AND MOtIVATION}

Spiking Neural Networks (SNNs) - These are computing paradigms with spiking neurons and synapses. A neuron fires a spike when its membrane voltage exceeds a threshold and subsequently the membrane voltage is reset. The moment of threshold crossing defines the firing time.

SNNs can be used to implement supervised machine learning approaches, where they are first trained with examples from the field and then deployed for inference with infield data. Performance is measured using accuracy, which is assessed from inter-spike intervals (ISIs) [9]. To define ISI, we consider a neuron excited with an input over some finite interval of time $[0, T]$. Neural activities in this time interval generate $K$ spikes with spike times $\left\{t_{1}, t_{2}, \cdots, t_{K}\right\}$. The average ISI of this spike train is given by [10]:

$$
\mathcal{I}=\left(\sum_{i=2}^{K} t_{i}-t_{i-1}\right) /(K-1) \text {. }
$$

Neuromorphic Architectures - Figure 1(a) shows a neuromorphic architecture with 6 crossbars interconnected using a time-shared interconnect. A crossbar is a two-dimensional arrangement with $n$ rows, $n$ columns, and NVM elements (to store synaptic weights) at every cross-point. Each crossbar can map at most $n$ synapses per neuron. We illustrate an example, where two charge pumps are used to power these 9 crossbars, i.e., supply bias voltages necessary to read and program NVM cells in these crossbars.

Figure 1(b) illustrates a small SNN with two pre-synaptic neurons (N1 and N2) connected to a post-synaptic neuron (N3), and their mapping to a crossbar. Synaptic weight $w_{13}$ is programmed on the NVM cell P1 and $w_{23}$ on P2. Output spike voltages $x_{1}$ from $\mathrm{N} 1$ and $x_{2}$ from N2 inject currents

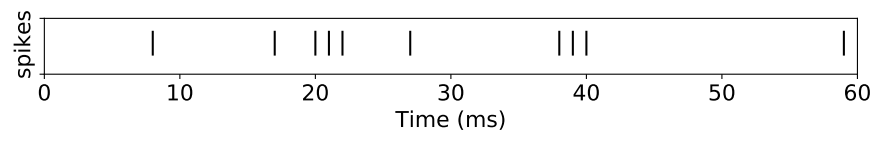

(a) Example spike train from N1 of Figure 1(b).

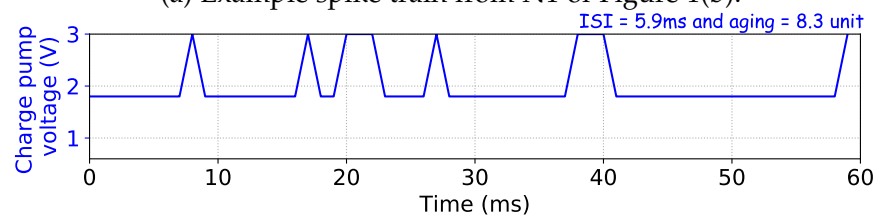

(b) Charge pump voltage to process the spike train.

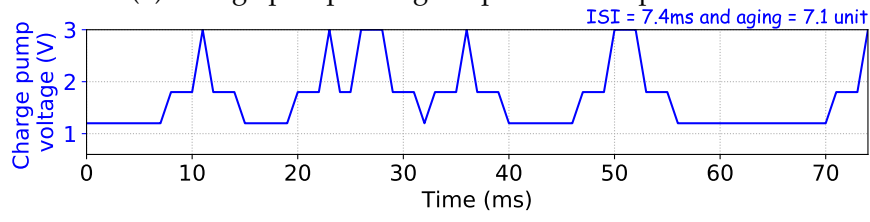

(c) Charge pump reset to $1.2 \mathrm{~V}$ after processing every spike.

Fig. 2: Illustrating the trade-off between charge pump aging and SNN performance, considering PCM crossbars.

into the crossbar, which are obtained by multiplying a presynaptic neuron's output spike voltage with the NVM cell's conductance at the cross-point of the pre- and post-synaptic neurons (following Ohm's law). Current summations along columns are performed in parallel using Kirchhoffs current law, and implement the sums $\sum_{j} w_{i j} x_{i}$, needed for forward propagation of neuron excitation $x_{i}$.

Figure 2(a) shows an example spike train from the presynaptic neuron (N1) of Figure 1(b). Each spike injects current to read the conductance of the NVM cell P1. Figure 2(b) illustrates the charge pump's operating voltage to process this spike train considering PCM crossbars. The charge pump is operated at $1.8 \mathrm{~V}$ for the entire $60 \mathrm{~ms}$ interval, boosting its voltage to $3 \mathrm{~V}$ only to process spikes. For this example, aging of the charge pump is 8.3 unit (see Section 3 for aging computation) and the average ISI is $5.9 \mathrm{~ms}$.

Figure 2(b) illustrates the charge pump's operating voltage when it is discharged to $1.2 \mathrm{~V}$ after processing every spike and boosted again to $1.8 \mathrm{~V}$ before processing the next. Once discharged, the crossbar becomes unavailable to process spikes, introducing latency in processing the spike train. The average ISI increases to $7.4 \mathrm{~ms}$, compared to $5.9 \mathrm{~ms}$ in Figure 2(b). ISI deviation leads to accuracy loss [11]. Frequently discharging the charge pump, however, reduces its aging to 7.1 unit, compared to 8.3 unit in Figure 2(b). Aging reduction improves the charge pump's lifetime.

\section{Proposed Workload-Aware Framework}

We briefly review the NBTI aging model and then present our framework for PCM crossbars. The framework can be extended for other NVMs such as FeRAM and Flash, where high voltage operations lead to aging issues. The framework can also model aging due to TDDB and HCI.

NBTI Aging Model - NBTI is one of the most critical reliability issues in silicon-based MOSFETs. It manifests itself as 1) decrease in drain current and transconductance, and 2) increase in off current and threshold voltage. NBTI aging is accelerated at high temperature and high oxide 
electric field. Recent works such as [7] suggest that NBTI is the collective response of two independent defects - the as-grown hole traps (AHTs) and generated defects (GDs). AHTs and a small proportion of GDs can be recovered by annealing at high temperatures if the NBTI stress voltage is removed. Our focus is on modeling GDs that form the permanent components. In fact, once introduced, GDs cannot be eliminated. Their growth can, however, be decelerated by applying lower voltages (viz. through discharging of stressed charge pumps).

To formulate NBTI aging, we divide the SNN execution time $[0, T]$ into $m$ equal intervals $0=t_{0}<t_{1} \cdots<t_{m}=T$, with $\left[t_{i}, t_{i+1}\right)$ as the $(i+1)^{\text {th }}$ interval and $V_{i}$ is the charge pump's voltage in this interval. Reliability at the end of SNN execution can be expressed as

$$
R(T)=e^{-\left(\sum_{i=0}^{m-1} G\left(V_{i}\right)\right)^{\beta}},
$$

where $\beta$ is the shape parameter of the NBTI defect distribution and $G\left(V_{i}\right)$ is the generated defect at voltage $V_{i}$, expressed as power law, $G\left(V_{i}\right)=g_{0} \cdot\left(V_{i}-V_{\text {th }}\right)^{m} \cdot\left(t_{i+1}-t_{i}\right)^{n}$, where $g_{0}, m, n$ are material-dependent constants [7]. We can then define aging in a stressed charge pump due to NBTI failures as

$$
\mathcal{A}=\sum_{i=0}^{m-1} g_{0} \cdot\left(V_{i}-V_{\text {th }}\right)^{m} \cdot\left(t_{i+1}-t_{i}\right)^{n} \text {, such that } R(T)=e^{-\mathcal{A}^{\beta}} .
$$

Here (3) assumes all synapses of the SNN to be mapped to the same crossbar, which is powered by a single charge pump. In practice, however, 1) synapses are distributed across different crossbars of the architecture because a crossbar can accommodate only a limited number of synapses and 2) a neuromorphic architecture integrates more than one charge pump to balance the power delivery to different crossbars. We now describe how to extend (3) to incorporate these practical constraints.

We consider the $\mathrm{SNN} \mathcal{G}$, with $N$ neurons and $S$ synapses, excited with an input over the time interval $[0, T]$. We arrange the spikes in this interval by synapses they excite as

$$
\mathcal{S}=\left\{\tau_{1}^{1}, \tau_{2}^{1}, \cdots, \tau_{k_{1}}^{1}\right\},\left\{\tau_{1}^{2}, \tau_{2}^{2}, \cdots, \tau_{k_{2}}^{2}\right\}, \cdots,\left\{\tau_{1}^{S}, \tau_{2}^{S}, \cdots, \tau_{k_{S}}^{S}\right\},
$$

where $\tau_{j}^{s}$ is the $j^{\text {th }}$ spike on $s^{\text {th }}$ synapse of the SNN. We introduce the following notation.

$$
\begin{aligned}
& \mathcal{A}_{s} \text { : aging to process spike train }\left\{\tau_{1}^{s}, \cdots, \tau_{k_{s}}^{s}\right\} \text { on } s^{\text {th }} \text { synapse } \\
& C \text { : number of crossbars } \\
& L \text { : number of charge pumps }
\end{aligned}
$$

$\mathcal{M} \in \mathbb{R}^{S \times C}$ :synapse-to-crossbar mapping, such that

$$
\begin{gathered}
m_{i j} \in \mathcal{M}= \begin{cases}1 & \text { if synapse } i \text { is mapped to crossbar } j \\
0 & \text { otherwise }\end{cases} \\
\mathcal{P} \in \mathbb{R}^{C \times L} \text { :crossbar-to-charge pump mapping, such that } \\
p_{j k} \in \mathcal{P}= \begin{cases}1 & \text { if crossbar } j \text { is powered by charge pump } k \\
0 & \text { otherwise }\end{cases}
\end{gathered}
$$

Combining these two equations, we generate the synapseto-charge pump mapping as

$$
m_{i j} \cdot p_{j k}= \begin{cases}1 & \text { if synapse } i \text { is powered by charge pump } k \\ 0 & \text { otherwise }\end{cases}
$$

The total aging of charge pump $k$ is therefore

$$
\operatorname{aging}_{k}=\sum_{i=1}^{S} \sum_{j=1}^{C} m_{i j} \cdot p_{j k} \cdot \mathcal{A}_{i}
$$

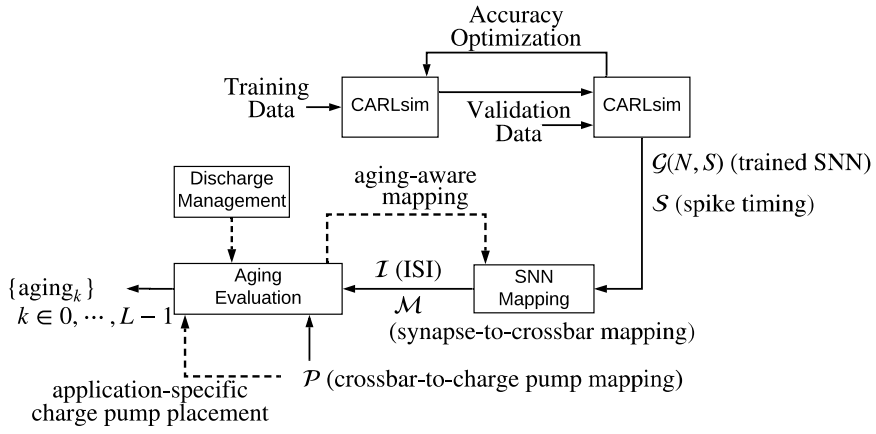

Fig. 3: Framework to evaluate aging of charge pumps.

Proposed Framework - Figure 3 illustrates our framework to evaluate aging of charge pumps in a neuromorphic architecture. We use the CARLsim simulator [8] to train a SNN using the training and validation data. Apart from the trained model, CARLsim also generates $\mathcal{S}$, the precise time of spikes on all synapses of the SNN. A SNN mapping approach such as [11] uses CARLsim output to generate the synapse-to-crossbar mapping $\mathcal{M}$, optimizing some objective function. In [11], the objective function is to minimize the number of spikes communicated between crossbars, which leads to lower energy and latency on the shared interconnect. Once the SNN is mapped to crossbars of the architecture, its performance is obtained in terms of the inter-spike interval $\mathcal{I}$ using (1). Using this synapse-tocrossbar and crossbar-to-charge pump mapping, our novel formulation in (8) evaluates the aging of all charge pumps in the architecture when executing the SNN workload. This design flow is shown using solid arrows.

Figure 3 also illustrates three future directions based on this framework using dashed arrows. First, Aging Evaluation, as developed in (8), can be combined with the SNN Mapping step to generate an optimum mapping of the SNN to the hardware that balances aging of all charge pumps. This is shown by the dashed arrow labeled aging-aware mapping. Second, crossbar-to-charge pump mapping can be optimized to achieve a desired lifetime of charge pumps for executing the SNN. This is shown using the dashed arrow labeled application-specific charge pump placement. Third, strategies can be developed to discharge charge pumps at run-time to improve their lifetime. This is shown in the Discharge Management step.

\section{Preliminary Evaluation}

This section presents some preliminary evaluation results using our framework. We use the neuromorphic architecture of Figure 1(a) to evaluate the following SNNs [12].

\begin{tabular}{c|cl|c}
\hline SNN & Synapses & Topology & Spikes \\
\hline ImgSmooth & 136,314 & FeedForward $(4096,1024)$ & 17,600 \\
EdgeDet & 272,628 & FeedForward $(4096,1024,1024,1024)$ & 22,780 \\
MLP-MNIST & 79,400 & FeedForward $(784,100,10)$ & $2,395,300$ \\
HeartEstm & 636,578 & Recurrent & $3,002,223$ \\
HeartClass & $2,396,521$ & $\mathrm{CNN}^{1}$ & $1,036,485$ \\
CNN-MNIST & 159,553 & $\mathrm{CNN}^{2}$ & 97,585 \\
LeNet-MNIST & $1,029,286$ & $\mathrm{CNN}^{3}$ & 165,997 \\
LeNet-CIFAR & $2,136,560$ & $\mathrm{CNN}^{4}$ & 589,953 \\
\hline 1. $(82 \times 82)-\left[\right.$ Conv, Pool] ${ }^{*} 16-\left[\right.$ Conv, Pool] ${ }^{*} 16-\mathrm{FC}^{*} 256-\mathrm{FC}^{*} 6$ \\
2. $(24 \times 24)-\left[\right.$ Conv, Pool] ${ }^{*} 16-\mathrm{FC}^{*} 150-\mathrm{FC}^{*} 10$ & \\
3. $(32 \times 32)-\left[\right.$ Conv, Pool] ${ }^{*} 6-\left[\right.$ Conv, Pool] ${ }^{*} 16-\mathrm{Conv}^{*} 120-\mathrm{FC}^{*} 84$ \\
4. $(32 \times 32 \times 3)-\left[\right.$ Conv, Pool] ${ }^{*} 6-\left[\mathrm{Conv}\right.$, Pool] ${ }^{*} 6-\mathrm{FC}^{*} 84-\mathrm{FC}^{*} 10$
\end{tabular}




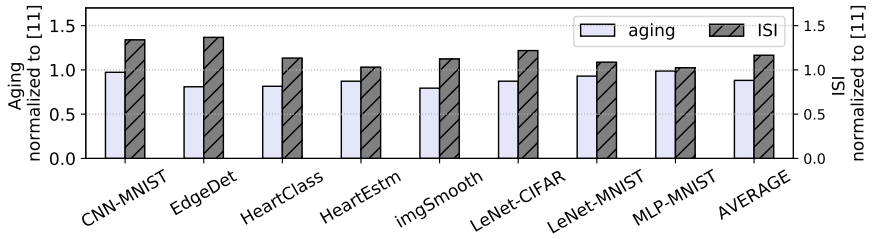

Fig. 4: Aging and ISI achieved by the aging-aware balancing approach compared to baseline [11].

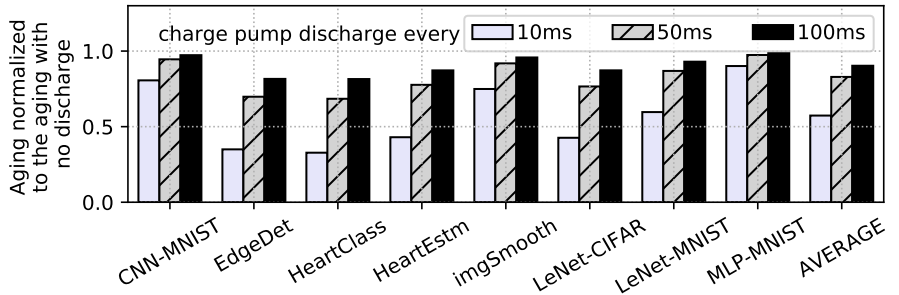

(a) Aging for different discharge intervals normalized to the aging when charge pumps are not discharged.

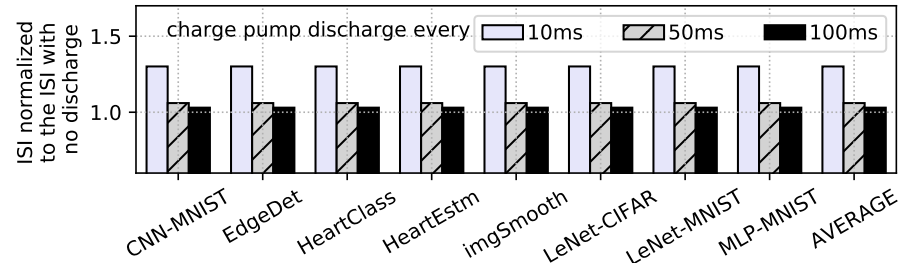

(b) ISI for different discharge intervals normalized to the ISI when charge pumps are not discharged.

Fig. 5: Aging and ISI with different discharge intervals.

\subsection{Aging-aware synapse placement}

We used the framework of Figure 3 to implement an agingaware balancing technique which distributes synapses to crossbars so as to balance the aging of all charge pumps in the architecture. Figure 4 plots the achieved ISI and aging for SNN workloads, normalized to [11] which performs no such balancing. Aging is reduced by an average of $12 \%$, improving lifetime of neuromorphic computing. This improvement, however, incurs an average of $16 \%$ increase in ISI, which leads to some performance degradation. Other techniques can be investigated using our framework, exploiting the lifetime and performance trade-off.

\subsection{Discharging stressed charge pumps}

Figure 5 illustrates aging and ISI with discharge intervals of $10 \mathrm{~ms}, 50 \mathrm{~ms}$, and $100 \mathrm{~ms}$ for the evaluated SNN workloads, normalized to when charge pumps are stressed for the entire execution duration. We make the following three key observations. First, aging is the lowest for discharge interval of $10 \mathrm{~ms}$, while ISI variation is the highest. Second, when the discharge interval changes from $10 \mathrm{~ms}$ to $100 \mathrm{~ms}$, aging increases, reducing charge pump's lifetime and ISI variation reduces, improving application performance. Third, aging of charge pumps varies across different SNN workloads. For MLP-MNIST, aging increases by $10 \%$ when the discharge interval increases from $10 \mathrm{~ms}$ to $100 \mathrm{~ms}$, while for LeNetCIFAR, aging increases by a factor of 2 for the same range. ISI variations are, however, comparable across different SNN workloads. Our framework enables exploration of SNN workload-specific lifetime and performance trade-offs.

\section{Conclusion and Future Outlook}

We proposed a novel framework to evaluate SNN workload-specific lifetime and performance trade-offs in neuromorphic architectures. The framework incorporates the CARLsim simulator to extract the precise time of spike generation on all synapses of an SNN workload. Using this timing information, together with 1) synapse-to-crossbar mapping, and 2) crossbar-to-charge pump mapping, this framework evaluates aging of different charge pumps when executing an SNN workload. We use this framework within an aging-aware synapse mapping technique and demonstrated an average of $12 \%$ reduction in aging and $16 \%$ increase in ISI compared to a state-of-the-art approach. We also demonstrated lifetime and performance trade-offs by changing the charge pump's discharge interval. Our framework can be easily extended to incorporate:

1. other wear-out formulations - We demonstrated our framework considering NBTI failures for PCM. Equation 3 can be extended to other failure mechanisms such as HCI and TDDB and for other NVMs such as FeRAM and Flash. 3. other SNN simulators - we used CARLsim, which allow modeling biological neurons and synapses and extracting precise spike timings. Our framework can be easily extended to consider other SNN simulators such as Brian [13] that allow extraction of precise spike timings.

4. other SNN mapping techniques - we evaluated one state-of-the-art SNN mapping [11]. Our framework can be used to evaluate other SNN mapping strategies, including those that target mapping SNNs to a single crossbar [14].

\section{REFERENCES}

[1] M. V. DeBole et al., "TrueNorth: Accelerating From Zero to 64 Million Neurons in 10 Years," IEEE Computer, 2019.

[2] M. Davies et al., "Loihi: A neuromorphic manycore processor with on-chip learning," IEEE Micro, 2018.

[3] S. Moradi et al., "A Scalable Multicore Architecture with Heterogeneous Memory Structures for Dynamic Neuromorphic Asynchronous Processors (DYNAPs)," IEEE Transactions on Biomedical Circuits and Systems, 2018.

[4] W. Maass, "Networks of spiking neurons: the third generation of neural network models," Elsevier Neural networks, 1997.

[5] G. W. Burr et al., "Neuromorphic computing using non-volatile memory," Advances in Physics: X, 2017.

[6] B. Shen and M. L. Johnston, "Zero reversion loss, high-ffficiency sharge pump for wide output current load range," in IEEE International Symposium on Circuits and Systems (ISCAS), 2018.

[7] R. Gao et al., "Nbti-generated defects in nanoscaled devices: fast characterization methodology and modeling," IEEE Transactions on Electron Devices, vol. 64, no. 10, pp. 4011-4017, 2017.

[8] T. Chou et al., "CARLsim 4: An open source library for large scale, biologically detailed spiking neural network simulation using heterogeneous clusters," in Intl. Joint Conf. on Neural Networks (IJCNN), 2018.

[9] D. P. Phillips and S. A. Sark, "Separate mechanisms control spike numbers and inter-spike intervals in transient responses of cat auditory cortex neurons," Elsevier Hearing research, 1991.

[10] S. Grün et al., Analysis of parallel spike trains. Springer, 2010.

[11] A. Das et al., "Mapping of local and global synapses on spiking neuromorphic hardware," in Design, Automation $\mathcal{E}$ Test in Europe Conference E Exhibition (DATE), 2018.

[12] MLPerf: Fair and useful benchmarks for measuring training and inference performance of ML hardware, software, and services. https://mlperf.org/training-overview/overview.

[13] D. F. Goodman and R. Brette, "The Brian Simulator," Frontiers in Neuroscience, vol. 3, p. 26, 2009.

[14] M. K. F. Lee et al., "A system-level simulator for RRAM-based neuromorphic computing chips," ACM Transactions on Architecture and Code Optimization (TACO), vol. 15, no. 4, p. 64, 2019. 